\title{
A COURSE ON SENSITIVITY ANALYSIS FOR GRADIENT ESTIMATION OF DES PERFORMANCE MEASURES
}

Felisa J. Vázquez-Abad

DIRO, Université de Montréal, C.P. 6128 Succursale Centre-Ville, H3C 3J7 Canada. Also Fellow, DEEE Melbourne University.

vazquez@IRO.UMontreal.CA

\section{Introduction}

Performance measures for stochastic Discrete Event Systems (DES) often involve finite or infinite horizon expectations of measurable costs and benefits. In its broader sense, the term "sensitivity analysis" refers to the estimation of the impact of changes in expected performance upon changes of some of the input parameters. In the particular case where the expected performance is differentiable, sensitivity analysis deals with the estimation of gradients of the expected performance with respect to some parameter of interest, called the control variable.

The past two decades have seen a fruitful period for developping sensitivity estimators for stochastic DES, and successful implementations include applications in telecommunications, manufacturing, finance, queueing and inventory systems. The rapid pace at which seemingly different methods have been proposed make it dificult even for experts to oversee their similarities and differences. The approach that we follow here differs somewhat from the texts available to date (see references) in that we propose a methodological study and explain how different estimation techniques can be "recovered". Selected examples will serve as the basis for the construction of the corresponding sensitivity estimators.

Sensitivity estimation can be divided in three categories: the pathwise analysis (covering the so-called perturbation analysis-or PA methods), the weak differentiation methodology, and perturbation methods, which include finite differences, harmonic analysis and simultaneous perturbations. This course focuses on the first two categories, opening with a review of the basic concepts of probability theory required in the sequel. 


\section{PROBABILITY CONCEPTS}

The building block for the mathematical description of a stochastic process is the concept of a measurable space, which defines the information structure of the model. It is customary to call $\Omega$ the space of states of nature. Given $\Omega$, the possible "observables" ${ }^{1}$ are subsets of $\Omega$. The appropriate information structure is that which includes all subsets of $\Omega$, and that is closed under countable unions and intersections.

Definition 1 A set $\mathfrak{F}$ of subsets of $\Omega$ is a $\sigma$-field if $\emptyset \in \mathfrak{F}, \Omega \in \mathfrak{F}$, for every $A \in \mathfrak{F}, A^{c} \in \mathfrak{F}$, and for any countable sequence of subsets $A_{n} \in \mathfrak{F}, n \in \mathbb{N}$, $\cup_{n} A_{n} \in \mathfrak{F}$.

Definition 2 Let $\mathfrak{F}$ be a $\sigma$-field on $\Omega$. A random variable is a function $X: \Omega \rightarrow \mathbb{R}$ that is measurable with respect to $\mathfrak{F}$, that is, if for any $x \in \mathbb{R}$, the set $\{\omega \in \Omega: X(\omega) \leq x\} \in \mathfrak{F}$.

Suppose that $\Omega=\left\{\omega_{1}, \ldots, \omega_{3}\right\}$ and that $\mathfrak{J}=\left\{\emptyset,\left\{\omega_{1}, \omega_{2}\right\},\left\{\omega_{3}\right\}, \Omega\right\}$. Then the function $X\left(\omega_{1}\right)=X\left(\omega_{3}\right)=1, X\left(\omega_{2}\right)=0$, for example, is not a random variable on $(\Omega, \mathfrak{F})$. In words, the information structure given by $\mathfrak{F}$ is not sufficient to describe the behaviour of the function $X$. Any real valued (bounded) function on $\Omega$ induces an information structure on $\Omega$ that makes it measurable, as stated in the following definition.

Definition 3 Given a function $X: \Omega \rightarrow \mathbb{R}$, the $\sigma$-field generated by $X$ is the minimal $\sigma$-field of $\Omega$ that contains the sets $\{\omega \in \Omega: X(\omega) \leq x\}$, for all $x \in \mathbb{R}$. It is denoted by $\sigma(X)$.

For the example above, $\sigma(X)=\left\{\emptyset,\left\{\omega_{1}, \omega_{3}\right\},\left\{\omega_{2}\right\}, \Omega\right\} \neq \mathfrak{F}$. If several random variables $X_{1}, \ldots, X_{n}$ are defined on a common probability space $(\Omega, \mathfrak{F})$, then $\sigma\left(X_{i}\right) \subset \mathfrak{F}$. The information structure generated by two or more random variables contains all the observables for all these variables, that is, $\sigma\left(X_{1}, \ldots, X_{n}\right)$ contains all the sets of the form $\left\{\omega \in \Omega: X_{i}(\omega) \leq\right.$ $\left.x_{i} ; i=1, \ldots, n\right\}$ for $x \in \mathbb{R}^{n}$.

Definition $4 A$ random process on $(\Omega, \mathfrak{F})$ is a sequence $X_{t}$ such that for each $t, X_{t}: \Omega \rightarrow \mathbb{R}$, and $\mathfrak{J}_{t} \equiv \sigma\left(X_{s} ; s \leq t\right) \subset \mathfrak{F}$. $A$ filtration is a sequence of increasing $\sigma$-fields on $\Omega$. In particular, $\mathbb{F}=\left(\mathfrak{\mho}_{t} ; t \geq 0\right)$ is called the natural filtration of the process.

Knowledge of the history of the process up to time $t$ is represented by the information structure given by $\mathfrak{f}_{t}$ : all random variables which are $\mathfrak{J}_{t}$-measurable can be expressed as functions of $\left(X_{s}, 0 \leq s \leq t\right)$.

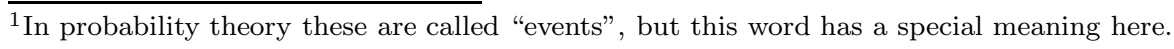


It follows from the above definitions that random processes can be defined without specifying a probability. The mathematical concept that captures the appropriate description of processes under uncertainty is that of a probability "measure". The idea that to each observable we must be capable of assigning a "likelihood" leads to the definition of the probability as a set function that takes elements from $\mathfrak{f}$ and assigns them a value in $[0,1]$.

Definition $5 A$ probability measure $\mathbb{P}$ on $(\Omega, \mathfrak{F})$ is a function $\mathbb{P}: \mathfrak{F} \rightarrow$ $[0,1]$ with $\mathbb{P}(\emptyset)=0, \mathbb{P}(\Omega)=1$ and such that for all countable collection of nonintersecting sets $\left(A_{n}, n \in \mathbb{N}\right) \in \mathfrak{F}$,

$$
\mathbb{P}\left(\bigcap_{n=1}^{\infty} A_{n}\right)=\sum_{n=1}^{\infty} \mathbb{P}\left(A_{n}\right) .
$$

More generally, a finite signed measure $\mu$ is a function $\mu: \mathfrak{f} \rightarrow \mathbb{R}$ with $\mu(\emptyset)=0, \sup _{A \in \mathfrak{Y}}\|\mu(A)\|<\infty$, satisfying the above additivity condition.

The model specifies an information structure $\mathfrak{F}$ on a given space of states of nature $\Omega$, endowed with a measure $\mathbb{P}$. Let $X$ be a random variable on $(\Omega, \mathfrak{F})$ and $\mathbb{P}$ a probability measure. Then $\mathbb{P}$ induces a likelihood function for the random variable $X$ as follows:

Definition 6 Let $X_{1}, \ldots, X_{n}$ be random variables on a common space $(\Omega, \mathfrak{F}, \mathbb{P})$, with state space $S \subset \mathbb{R}^{n}$. The probability induced by a random vector $X \in S$ is a measure on $(S, \mathcal{B})$, given by: $\mathrm{P}(X \in B)=\mathbb{P}\{\omega$ : $X(\omega) \in B\}$, for all $A \in \mathcal{B}$, where $\mathcal{B}$ contains all the sets $B$ for which $\{\omega: X(\omega) \in B\} \in \mathfrak{F}$. The distribution function of $X$ is the function $F_{X}: \mathbb{R}^{n} \rightarrow[0,1]$ defined for each $x \in \mathbb{R}^{n}$ by

$$
F_{X}(x)=\mathbb{P}\{\omega \in \Omega: X(\omega) \leq x\} .
$$

When $\mathcal{B}$ contains all hypercubes of the form $-a_{i}<x_{i} \leq b_{i}, i=1, \ldots n$, it is called the "Borel field" of $\mathbb{R}^{n}$, denoted $\mathcal{B}\left(\mathbb{R}^{n}\right)$.

Statistical analyses that depend only on the distribution function do not require explicit representation in terms of the "original" space of the states of nature $\Omega$. Suppose that a state space $S \subset \mathbb{R}^{n}$ endowed with a $\sigma$-field $\mathcal{B}$ is given, and that $\mathrm{P}$ is a measure defined on $(S, \mathcal{B})$. Then the distribution function is $\left.F(x)=\mathrm{P}\left\{\left(-\infty, x_{1}\right] \times \cdots \times\left[-\infty, x_{n}\right]\right)\right\}$, for $x \in S$, and it corresponds to a random vector $X \in S$. It is often the case that saying " $X$ is a random variable with exponential distribution of intensity $\theta$ " suffices to model a particular situation: here $F(x)=1-e^{-\theta x}$ and the actual set $\Omega$ becomes irrelevant. 
Modeling in Engineering and other Applied Sciences usually starts with this setting and considerable information can be obtained without the need for defining what the "states of nature" are; actually, many possibilities for $(\Omega, \mathfrak{F})$ exist which will reproduce the same distribution $F$ on $S$.

REMARK: It is possible to extend these concepts when $S$ is a more general space ${ }^{2}$. A distribution function can be defined using the appropriate topology for open intervals and it corresponds to a random entity $X \in S$. When $S$ is a space of functions of the form $f(t) \in \mathbb{R}, t \in[0, T)$, for each value $\omega, X(\omega)$ represents a whole trajectory of a dynamic process.

The "likelihood ratio", the "weak derivative" and the system perturbation estimators of the sensitivities are built directly on the functional space $(S, \mathcal{B})$. In contrast, all the methods for sensitivity analysis known under the generic name of "perturbation analysis" require an explicit construction of $\Omega$ and build the random processes as measurable functions $X(\omega)$. Representations are not unique, and as will be discussed, this plays an essential rôle in the construction of the estimators.

Theorem 1 (Skorohod Representation) Given a measurable random function $X: \Omega \rightarrow S$ on $(\Omega, \mathfrak{N}, \mathbb{P})$, there exists a function $\tilde{X}:[0,1] \rightarrow$ $S$ on $([0,1], \mathcal{B}([0,1]), \tilde{\mathbb{P}})$, where $\tilde{\mathbb{P}}$ is the Lebesgue measure (or uniform distribution on $[0,1]$ ), such that $X \stackrel{\mathcal{L}}{=} \tilde{X}$ (equality in distribution).

Let $S \subset \mathbb{R}$, and define the inverse distribution function of $F$ as $F^{-1}(u)=\min (x \in S: F(x) \geq u), u \in[0,1]$. It is well known that to generate a random variable with distribution $F$ it suffices to define $X(U)=F^{-1}(U)$, where $U \sim U[0,1]$ is a uniform random variate. In the case of the exponential distribution, $X(U)=-\frac{1}{\theta} \ln (1-U)$ is a random variable on $([0,1], \mathcal{B}([0,1]), \tilde{\mathbb{P}})$ having distribution $F(x)=1-e^{-\theta x}$, but so is the function $-\frac{1}{\theta} \ln (U)$, because both $U$ and $1-U$ have uniform distribution on the unit interval, which shows that the representation is not unique.

Expectations of a random variable $X$ on $(\Omega, \mathfrak{F})$ with respect to a probability measure $\mu$ are defined via the concept of Riemann integration.

Definition 7 The expected value of a random variable $X$ on $(\Omega, \mathfrak{F}, \mu)$, denoted by $\mathrm{E}[X]$, is defined as:

$$
\mathrm{E}[X]=\int_{\Omega} X(\omega) \mu(d \omega)=\sup _{\pi(\Omega)} \sum_{i}\left[\inf _{\omega \in A_{i}} X(\omega)\right] \mu\left(A_{i}\right),
$$

\footnotetext{
${ }^{2}$ Some technical assumptions must hold, such as the requirement that $S$ be a Polish space.
} 
(if this limit exists) where $\pi(\Omega)$ are all finite partitions of $\Omega:\left\{A_{i}, i=\right.$ $1, \ldots, N\}$, with $A_{j} \cap A_{j}=\emptyset$ for $i \neq j$.

For random variables that take only a countable number of values the above definition recovers the usual expression $\sum_{i} x_{i} \mathrm{P}\left[X=x_{i}\right]$. For continuous distributions, $\mathrm{E}[X]=\int x f(x) d x$, where $f(x)$ is the corresponding density function, and the sums represent the usual approximations to the integral. Equivalently, Skorohod representation can be used to define all expectations in terms of Lebesgue integration.

Definition 8 Given an observable set $A \in \mathfrak{F}$, with $\mathbb{P}(A)>0$, the conditional probability $\mathbb{P}(\cdot \mid A)$ is defined by $\mathbb{P}(B \mid A)=\mathbb{P}(B \cap A) / \mathbb{P}(A)$, for all $B \in \mathfrak{F}$.

Let $X$ be the random variable on $(\Omega, \Re, \mathbb{P})$ given by $X(\omega)=\mathbf{1}_{\{A\}}(\omega)$, then the information structure described by the knowledge of $X$ is $\sigma(X)=\left\{\emptyset, \Omega, A, A^{c}\right\}$ and the conditional probability satisfies $\mathbb{P}(\cdot \mid X=$ $1)=\mathbb{P}(\cdot \mid A)$. Analogously, $\mathbb{P}(\cdot \mid X=0)=\mathbb{P}\left(\cdot \mid A^{c}\right)$. The extended definition of the conditional probability accounts for the fact that for every $B \in \mathfrak{F}, \mathbb{P}(B \mid X)$ is a random variable. In particular this random variable takes the values $\mathbb{P}(B \mid A)$ for all $\omega \in A$ (for which $X(\omega)=1$ ) and $\mathbb{P}\left(B \mid A^{c}\right)$ for all $\omega \notin A$. In addition, $\mathbb{P}(B \mid X)$ is necessarily measurable with respect to $\sigma(X)$. Notice that the same holds true if $X(\omega)=$ $c \mathbf{1}_{\{A\}}(\omega)$ for any constant $c$. These concepts capture the essential properties of conditional expectations, which we state in the general case.

Definition 9 Let $X$ be a random variable on $(\Omega, \mathfrak{F}, \mathbb{P})$ and $\mathcal{G} \subset \mathfrak{F}$ a sub $\sigma$-field of $\Omega$. The conditional expectation $\mathrm{E}[X \mid \mathcal{G}]$ is a $\mathcal{G}$-measurable random variable $Z$ such that for all $B \in \mathcal{G}, \mathrm{E}\left(Z \mathbf{1}_{\{B\}}\right)=\mathrm{E}\left(X \mathbf{1}_{\{B\}}\right)$.

If $Y$ is any random vector on $(\Omega, \mathfrak{F}, \mathbb{P})$ satisfying $\sigma(Y)=\mathcal{G}$, then $\mathrm{E}[X \mid Y]=\mathrm{E}[X \mid \mathcal{G}]$.

Definition 10 A stochastic process $\left\{X_{n}, n \in \mathbb{N}\right\}$ with state space $S$ on $\left(\Omega, \mathbb{F}=\left\{\mathfrak{F}_{n}, n \in \mathbb{N}\right\}, \mathbb{P}\right)$ is called a Markov Chain if $X_{n}$ is $\mathfrak{F}_{n}$-measurable and if for every $A \subset S$

$$
\mathbb{P}\left(\left\{\omega: X_{n+1}(\omega) \in A\right\} \mid \Re_{n}\right)=\mathbb{P}\left(\left\{\omega: X_{n+1}(\omega) \in A\right\} \mid X_{n}\right),
$$

with probability 1. It is called a homogeneuos Markov Chain if the above probabilities are independent of $n$.

Use now the induced probability on the space $(S, \mathcal{B}(S))$, to write the familiar formula $\mathrm{P}\left(X_{n+1} \in A \mid \mathfrak{\jmath}_{n}\right)=\mathrm{P}\left(X_{n+1} \in A \mid X_{n}\right)$ for the onestep transition probabilities. For a homogeneous chain, given any $x \in S$, $p(x, \cdot)=\mathrm{P}\left(X_{n+1} \in A \mid X_{n}=x\right)$ is a probability on $\mathcal{B}(S)$. 
A discrete event system is a stochastic process with a state space $S \subset \mathbb{R}^{n}$ and a set of $d$ event-timing clocks $\mathcal{T}=\left(\mathbb{R}^{+}\right)^{d}$. The event space $E$ contains $d$ elements and for each $x \in S$ a set of possible events $\Gamma(x) \subset E$ is given. For $z=(x, y) \in S \times \mathcal{T}$, define $e(x, y)=\arg \min \left\{y_{i}: i \in \Gamma(x)\right\}$ as the the next event function, with $\tau(z)=Y_{e(z), t}$ the elapsed time.

Definition $11 A$ Discrete Event Process $\left\{Z_{t}(\theta)\right\}$ is a stochastic process on $S \times \mathcal{T}$. For $t \in \mathbb{R}^{+}, Z_{t}(\theta)$ represents the state of the physical process $X_{t}$ and the values of the residual clocks $Y_{t}$ for the events lifetimes, with:

$$
\begin{aligned}
& \operatorname{Prob}\left[X_{t+\tau} \in A \mid Z_{t}=(x, y)\right]=p_{\theta}(A ; x, e(x, y)), \quad A \in \mathcal{B}(S) \\
& Y_{i, t+\tau}=\left(Y_{i, t}-\tau\right) \mathbf{1}_{\left\{i \neq e\left(Y_{t}\right), i \in \Gamma\left(X_{t}\right)\right\}} \\
& \operatorname{Prob}\left[Y_{e\left(Z_{t}\right), t+\tau} \in B \mid Z_{t}=(x, y)\right]=\mathbb{G}_{e\left(Z_{t}\right), \theta}(B, x), \quad B \in \mathcal{B}(\mathcal{T})
\end{aligned}
$$

where, for $i \in E, x \in S, p_{\theta}(\cdot, x, i)$ is a probability measure on $\mathcal{B}(S)$, and $\mathbb{G}_{i, \theta}(\cdot, x)$ is a probability measure on $\mathcal{B}\left(\mathbb{R}^{+}\right)$. The variable $\theta \in \mathbb{R}^{s}$ is the control variable and represents several parameters of the distribution and process dynamics.

It follows that the embedded process $\left\{Z_{n}(\theta)\right\}$ observed only at the event epochs is a Markov chain in a general state space, also called a Generalized Semi-Markov Process. Use the probability space $(\Omega, \mathbb{F}=$ $\left.\left\{\mathfrak{\mho}_{t} ; t \geq 0\right\}, \mathbb{P}\right)$ under Skorohod representation of the DES $\left\{Z_{t}(\theta)\right\}$, where $\mathbb{P}$ is the uniform measure on the unit interval $\Omega$ (and thus is independent of $\theta$ ) and $\mathbb{F}$ is the natural filtration of the process.

Definition 12 The performance measure of a controlled DES is of the form $J(\theta)=\mathrm{E}[L(\theta)]$, and the sample performance is a function of the whole trajectory, that is, for each $\omega \in \Omega, L(\theta, \omega)=\phi\left(\theta, X_{t}(\theta, \omega) ; 0 \leq t \leq\right.$ $T$ ). The horizon $T$ can be finite, a random stoping time ( $a \mathfrak{\mho}_{t}$-measurable random variable), or infinite.

In practice, DES are used to model systems for which the designer can choose the value of the parameter $\theta$, or it can be adjusted dynamically through built-in controllers. In either case, one is interested in estimating the sensitivities of the expected performance $J(\theta)$ to changes in $\theta$. These sensitivities are often expressed in terms of the gradient $\nabla_{\theta} J(\theta)$. The problem of sensitivity estimation is the following. For most realistic models, a closed expression of $J(\theta)$ is unavailable and simulations of the system (or observations of several trajectories) have to be made in order to evaluate a sample average. This is the principle underlying Monte-Carlo simulation, because under the canonical representation:

$$
J(\theta)=\int_{\Omega} L(\theta, \omega) \mathbb{P}(d \omega)=\mathrm{E}\left[\frac{1}{N} \sum_{i=1}^{N} L\left(\theta, U_{i}\right)\right],
$$


where $U_{i}$ are independent uniform random variables. If $J(\theta)$ is not available in closed form, then neither is its gradient and the question is how to build a random vector $\hat{J}(\theta)$, called the sensitivity estimator such that $\mathrm{E}[\hat{J}(\theta)]=\nabla_{\theta} J(\theta) .{ }^{3}$ Sometimes it is possible to construct a "derivative process" on the same probability space where $Z_{t}(\theta)$ is defined, but in most cases it is necessary to enlarge the state space and the filtration to build the estimator. All available methods rely on direct observation of the DES process along its trajectories and the estimators are also functions of the path $\left(X_{t}(\theta, \omega) ; 0 \leq t \leq T\right)$. Yet the problem has been stated in terms of the expectations of the estimator. The following concepts establish the relationship between equality in distribution and pathwise equality.

Definition 13 Two random variables $X, Y \in S$ defined on a common probability space $(\Omega, \mathfrak{F}, \mathbb{P})$ are equal with probability 1 (w.p.1) or equal almost surely (a.s.) if $\mathbb{P}\{\omega: X(\omega)=Y(\omega)\}=1$.

They are said to be equal in distribution (denoted $X \stackrel{\mathcal{L}}{=} Y$ ) if $F_{X}(x)=$ $F_{Y}(x)$. Accordingly, if $\left(X_{n}, n \in \mathbb{N}\right)$ is a sequence of random variables on $(\Omega, \mathfrak{F}, \mathbb{P})$, and $X$ is $\mathfrak{\gamma}$-measurable,

- $X_{n}$ converges to $X$ almost surely, denoted by $X_{n} \rightarrow X$ a.s., if $\mathbb{P}\left\{\omega: \lim _{n \rightarrow \infty} X_{n}(\omega)=X(\omega)\right\}=1$.

- $X_{n}$ converges to $X$ weakly, denoted by $X_{n} \stackrel{\mathcal{L}}{\Longrightarrow} X$, if for every point $x \in S$ of continuity of $F_{X}, \lim _{n \rightarrow \infty} F_{X_{n}}(x) \rightarrow F_{X}(x)$.

Convergence in distribution is equivalent to the condition that for all bounded and continuous function $f: S \rightarrow \mathbb{R}, \mathrm{E}\left[g\left(X_{n}\right)\right] \rightarrow \mathrm{E}[g(X)]$. It is possible that $X_{n} \rightarrow X$ a.s. and yet $\mathrm{E}\left[X_{n}\right] \not \rightarrow \mathrm{E}[X]$. The classical example is given by the sequence $X_{n}(\omega)=n \mathbf{1}_{\{\omega<1 / n\}}$, on the canonical space $([0,1], \mathcal{B}([0,1]), \mathbb{P})$. The pathwise limit $X(\omega)=0$ for every $\omega>0$, and $\mathbb{P}[\omega=0]=0$, so $X \equiv 0$ is the a.s. limit of the sequence. Alas, $\mathrm{E}\left[X_{n}\right]=1$ for all $n \geq 1$, so $X_{n}$ does not converge in distribution.

Theorem 2 (Dominated Convergence) Let $\left(X_{n}, n \in \mathbb{N}\right), X$ be random variables on a common probability space $(\Omega, \mathfrak{F}, \mathbb{P})$. If $X_{n} \rightarrow X$ a.s. and there is a random variable $K$ on $(\Omega, \mathfrak{F}, \mathbb{P})$ such that $\mathrm{E}(K)<\infty$ and $\left|X_{n}\right| \leq K$ a.s. for all $n \in \mathbb{N}$, then $\lim _{n \rightarrow \infty} \mathrm{E}\left[X_{n}\right]=\mathrm{E}[X]$.

${ }^{3}$ For lack of space, the subject of unbiased, consisitent and strongly consistent estimation will not be explicitly dealt with here, but they are essential to sensitivity analysis. 


\section{PATHWISE ANALYSIS}

In the sequel, to simplify the exposition, consider $\theta \in \Theta \subset \mathbb{R}$ and let $\left\{Z_{t}(\theta), t \geq 0\right\}$ be a controlled DES. The pathwise analysis states the problem in terms of estimating

$$
J^{\prime}(\theta)=\frac{d}{d \theta} \int_{\Omega} L(\theta, \omega) \mathbb{P}(d \omega),
$$

where $L(\theta, \omega)=\phi\left(\theta, X_{t}(\theta, \omega) ; 0 \leq t \leq T\right)$ is the sample performance, defined on a probability space $(\Omega, \mathbb{P})$ independent of $\theta$. Small perturbations of $\theta$ are studied for each trajectory $\omega$ to estimate the above sensitivity.

It is customary to assume that between consecutive events the physical state remains constant, and $S$ is assumed to be countable. However, there is no particular reason why the model should exclude some interesting problems where the trajectories of $X_{t}(\omega)$ are continuously differentiable between event occurrences. Our first example deals with such a system, which we view as a discrete event driven process.

\section{EXAMPle 1. Ruin Probabilities.}

The surplus process of an insurance portfolio is defined as the wealth obtained by the premium payments at constant rate $c$, minus the reimboursements made at the times of claims. The claim arrival process $\{N(t) ; t \geq 0\}$ is Poisson with intensity $\theta$ and claims are i.i.d random variables $\left\{Y_{i}\right\} \sim G$, with $\beta=\mathrm{E}\left[Y_{i}\right]$. It is assumed that the wealth available is invested at some continuously compounded interest rate $\delta$. The initial endowment of the company is $u$, and the surplus process satisfies:

$$
d U(t)=[c+\delta U(t)] d t-d S(t) ; \quad U(0)=u
$$

where $S(t)=\sum_{i=1}^{N(t)} Y_{i}$ is a compound Poisson process. Use the notation $\left\{T_{n}, n \geq 0\right\}$ for the event epochs of the process $N(t)$, with $T_{0} \equiv 0$, and denote by $W_{n}=T_{n}-T_{n-1}$ the interarrival times. Set $n^{*}=\min \{n$ : $\left.U\left(T_{n}\right)<0\right\}$, then the ruin probability is $J(\theta)=P\left\{n^{*}<\infty\right\}$ and it is a measure of the credit risk of the company. It is well known in actuarial science that there is a duality between the surplus process and a storage process that generalizes the concept of work in a queueing system, defined by: $X(t)=d S(t)-(c+\delta X(t))^{+} d t$, where $(x)^{+} \equiv \max (0, x)$, and the ruin probability is equal to the overflow probability:

$$
J(\theta)=\lim _{t \rightarrow \infty} P\{X(t)>u\}=\lim _{t \rightarrow \infty} \frac{1}{t} \sum_{n=1}^{N(t)} D_{n},
$$

where $D_{n}$ is the total amount of time that the process $X(\cdot)$ spends above level $u$ within the interval $\left[T_{n-1}, T_{n}\right)$. As shown in Figure 1, for 
$t \in\left[T_{n-1}, T_{n}\right)$ the process $X(t)$ follows an ODE and therefore:

$$
\begin{gathered}
X\left(T_{n-1}+s\right)=\left(e^{-\delta s}\left[X\left(T_{n-1}\right)-\frac{c}{\delta}\left(e^{\delta s}-1\right)\right]\right)^{+}, \quad s \in\left[0, W_{n}\right) \\
X\left(T_{n}\right)=\left(e^{-\delta W_{n}}\left[X\left(T_{n-1}\right)-\frac{c}{\delta}\left(e^{\delta W_{n}}-1\right)\right]\right)^{+}+Y_{n} .
\end{gathered}
$$

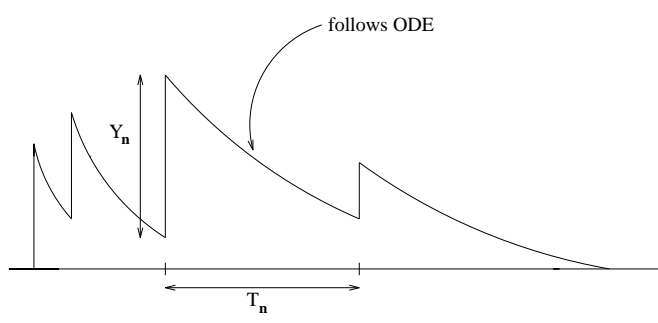

Figure 1 A cycle of $X(t)$.

The embedded process $\left\{X\left(T_{n}\right) ; n=0,1, \ldots\right\}$ can be used for discrete event simulation in order to estimate the sensitivity of the ruin probability. Abusing notation, use $\left\{X_{n}, n \in \mathbb{N}\right\}$ to denote the embedded chain.

This process regenerates at the event epochs $T_{i}$ when $X_{i}=0$. Call $\tau$ the first index such that $X_{\tau+1}=0$. The length of a regeneration cycle is $T_{\tau}$ and it is a.s. finite when $c>\theta \beta$ even if $\delta=0$. The regenerative formula establishes that in this case:

$$
J(\theta)=\frac{\mathrm{E}\left[\sum_{n=1}^{\tau} D_{n}\right]}{\mathrm{E}\left[T_{\tau}\right]},
$$

which yields:

$$
\frac{\partial}{\partial \theta} J(\theta)=\frac{1}{\mathrm{E}\left[T_{\tau}\right]}\left\{\frac{\partial}{\partial \theta} \mathrm{E}\left[\sum_{n=1}^{\tau} D_{n}\right]-\frac{\mathrm{E}\left[\sum_{n=1}^{\tau} D_{n}\right]}{\mathrm{E}\left[T_{\tau}\right]} \frac{\partial \mathrm{E}\left[T_{\tau}\right]}{\partial \theta}\right\},
$$

so the problem is to estimate both $\frac{\partial}{\partial \theta} \mathrm{E}\left[T_{\tau}\right]$ and $\frac{\partial}{\partial \theta} \mathrm{E}\left[\sum_{n=1}^{\tau} D_{n}\right]$.

Choosing the representation: Pathwise analysis requires expressing the sample perfomance as an explicit function of $\omega$. The choice that is usually made for all the PA methods (although it is not the only choice) is to write the Skorohod representation for each timing lifetime. The resulting representation of each trajectory over one cycle $(n<\tau)$ is:

$$
\begin{aligned}
& W_{n}(\theta)=-\frac{1}{\theta} \log \left(1-U_{n}\right), \quad U_{n} \sim U[0,1] \\
& X_{n+1}(\theta)=\left(e^{-\delta W_{n+1}(\theta)}\left[X_{n}(\theta)-\frac{c}{\delta}\left(e^{\delta W_{n+1}(\theta)}-1\right)\right]\right)+Y_{n+1} \\
& r_{n+1}(\theta)=\frac{1}{\delta} \ln \left[\frac{X_{n}(\theta)+c / \delta}{u+c / \delta}\right] \\
& D_{n+1}= \begin{cases}0 & \text { if } X_{n}(\theta) \leq u \\
\min \left(W_{n+1}(\theta), r_{n+1}(\theta)\right) & \text { otherwise. }\end{cases}
\end{aligned}
$$


The resulting model corresponds to a the sequence of independent random variables $\left\{\left(U_{n}, Y_{n}\right) ; n=1, \ldots, \tau\right\}$ that define a stochastic process on a probability space $(\Omega, \mathbb{P})$ which is indepedent of $\theta$. Notice that $\tau$ is a stopping time: knowledge of $\left\{\left(U_{i}, Y_{i}\right), i \leq n\right\}$ is sufficient to establish whether $\tau \leq n$ or not, so $\tau$ is $\mathfrak{J}_{\tau}$-measurable. All the dependency is expressed pathwise through the recursions above.

Pathwise derivatives: Because $T_{\tau}=\sum_{n=1}^{\tau} W_{n}(\theta)$, it follows that $\frac{\partial}{\partial \theta} T_{\tau}=-T_{\tau} / \theta$. For each $D_{n}$,

$$
\frac{\partial}{\partial \theta} D_{n}= \begin{cases}0 & \text { if } X_{n}(\theta) \leq u \\ -\frac{W_{n+1}(\theta)}{\theta} & \text { if } X_{n}(\theta)>u \text { and } W_{n+1}(\theta)<r_{n}(\theta) \\ \frac{\partial}{\partial \theta} r_{n}(\theta) & \text { if } X_{n}(\theta)>u \text { and } W_{n+1}(\theta)>r_{n}(\theta)\end{cases}
$$

where the derivative of $\frac{\partial}{\partial \theta} r_{n}(\theta)$ can be calculated recursively using the chain rule via the process $X_{n}(\theta)$. This can be used to obtain the sample derivatives of $L_{1}(\theta)=T_{\tau}$ and $L_{2}(\theta)=\sum_{n=1}^{\tau} D_{n}$.

\%

In order to use a pathwise derivative $L^{\prime}(\theta, \omega)$ (called the IPA derivative) as an estimator of $J^{\prime}(\theta)$, one must now answer the question: is $\mathrm{E}\left[L^{\prime}(\theta)\right]=\frac{\partial}{\partial \theta} \mathrm{E}[L(\theta)]=J^{\prime}(\theta)$ ? As stated in the Dominated Convergence Theorem, this is not always the case.

Definition 14 A deterministic function $L(\theta): \Theta \rightarrow S$ is called Lipschitz conintuous if there is a (Lipschitz) constant $K<\infty$ such that

$$
\sup _{\theta \in \Theta: \theta+\Delta \theta \in \Theta}\|L(\theta+\Delta \theta)-L(\theta)\| \leq K \Delta \theta,
$$

where the space $S$ is endowed with the norm $\|\cdot\|$.

$A$ random variable $L(\theta, \omega)$ on $(\Omega, \mathfrak{F}, \mathbb{P})$ is said to be a.s. Lipschitz continuous in $\theta$ if there is a measurable random variable $K<\infty$ with $\mathrm{E}[K]<\infty$ such that for each $\omega, L(\theta, \omega)$ is Lipschitz conintuous with Lipschitz constant $K(\omega)$.

Theorem 3 (Lipschitz Continuity) If $L(\theta)$ is a.s. Lipschitz continuous in $\theta$, then $\mathrm{E}\left[L^{\prime}(\theta)\right]=J^{\prime}(\theta)$.

Te proof is a straightforward application of the Dominated Convergence Theorem, together with the assumption that the limit:

$$
\lim _{\Delta \theta \rightarrow 0} \frac{J(\theta+\Delta \theta)-J(\theta)}{\Delta \theta}=J^{\prime}(\theta)
$$

exists and is uniformly bounded on the compact set $\Theta$. 
Example 1. Ruin Probabilities (continued).

In order to establish (or disproof) Lipschitz continuity, the pathwise analysis considers a single trajectory of the underlying process $\left\{\left(U_{n}, Y_{n}\right)\right\}$, and two paths are evaluated in parallel: one with control value $\theta$, often called the nominal path and another with control value $\theta+\Delta \theta$, called the perturbed path.

For $L_{1}(\theta)=\sum_{n=1}^{\tau} W_{n}(\theta)$ the analysis is simple:

$L_{1}(\theta+\Delta \theta, \omega)-L_{1}(\theta, \omega)=-\sum_{n=1}^{\tau} \delta_{n}(\theta), \quad \delta_{n}(\theta)=W_{n}(\theta+\Delta \theta)-W_{n}(\theta)$,

which holds for "sufficiently small" perturbations. This follows from the fact that $W_{n}(\theta+\Delta \theta)<W_{n}(\theta)$ a.s., and a constant $C$ exisits with $X_{n}(\theta+\Delta \theta)-X_{n}(\theta) \leq C\left(\delta_{1}+\ldots+\delta_{n}\right)$. Given the trajectory at $\theta$, it is then possible to choose a sufficiently small $\Delta \theta$ so that the cycles finish at the same iteration $\tau$. Next, a constant $K$ can be found using a Taylor expansion to establish that $\left|\delta_{n}(\theta)\right| \leq K W_{n}(\theta) \Delta \theta$, so that $L_{1}(\theta)$ is a.s. Lipschitz continuous because the Lipschitz constant is bounded by $K T_{\tau} \Delta \theta$, and $\mathrm{E}\left[T_{\tau}\right]<\infty$.

Propagation of the perturbations: For $L_{2}(\theta)$ there are two sources of possible discontinuities for each $D_{n}(\theta)$ : level changes, when $X_{n}(\theta)>$ $u>X_{n}(\theta-\Delta \theta)$ (illustrated to the left in Figure 2) and residual time changes (illustrated to the right in Figure 2), when $W_{n}(\theta)<r_{n}(\theta)$ but $W_{n}(\theta-\Delta \theta)>r_{n}(\theta-\Delta \theta)$. Studying each trajectory in detail, it can be shown that $\sum_{n=1}^{\tau} D_{n}(\theta)$ is indeed a.s. Lipschitz continuous, provided now that $\mathrm{E}\left[T_{\tau}^{2}\right]<\infty$.

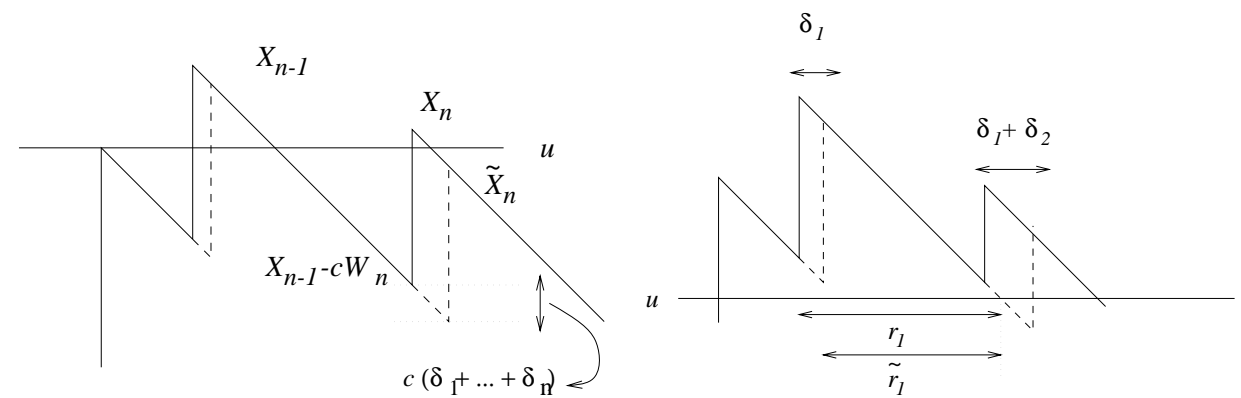

Figure 2 Sources of path discontinuiuties.

Continuous flow analysis: Now let us rewrite:

$$
L_{2}(\theta, \omega)=\int_{0}^{\tau} \mathbf{1}_{\left\{X_{t}(\theta, \omega)>u\right\}} d u,
$$


where we have used the continuous time representation of the process. An alternative to the detailed pathwise analysis is the study of piecewise continuous functions. The trajectory $X(\cdot, \omega)$ is not Lipschitz continuous in the sup-norm, that is, $\|X(\theta)-X(\theta-\Delta \theta)\|_{\infty}=\sup _{0 \leq s \leq T_{\tau}} \mid X_{s}(\theta, \omega)-$ $X_{s}(\theta-\Delta \theta, \omega) \mid$ is as large as the maximal claim amount in the cycle. However, it is Lipschitz continuous in $L^{1}$-norm, that is, for fixed $\omega$,

$\|X(\theta)-X(\theta-\Delta \theta)\|_{1}=\int_{0}^{T_{\tau}}\left|X_{s}(\theta)-X_{s}(\theta-\Delta \theta)\right| d s \leq \max _{n \leq \tau}\left(Y_{n}\right) K T_{\tau} \Delta \theta$.

The continuous flow analysis studies each trajectory as a piecewise differentiable function to establish continuity of $L_{2}(\theta)$, that is, if $X_{t}, Y_{t}$ are such that $\|X-Y\|_{1} \leq K \Delta \theta$ then the corresponding sample performances differ only in an amount which is linear in $\Delta \theta$.

In summary, the IPA estimators $L_{1}^{\prime}(\theta)$ and $L_{2}^{\prime}(\theta)$ are unbiased if $\mathrm{E}\left[\max _{n \leq \tau}\left(Y_{n}\right) T_{\tau}\right]<\infty$. This condition is somewhat stronger than the condition obtained through the detailed analysis of the propagation of the perturbations, which holds true if $\mathrm{E}\left[Y_{1}^{2}\right]<\infty$.

REMARK: Even if $X(\theta)$ is a.s. Lipschitz continuous w.r.t. $\theta$ (in the $L^{1}$-norm), the sample performance may fail to be so. Consider evaluating the derivative of $\mathrm{E}(\tau)$; the pathwise derivative is identically zero.

\%*

Suppose that Skorohod representation has been chosen for either the clock or state variables-depending on which distribution is affected by the control variable $\theta$. Under this representation, $L(\theta, \omega)$ may fail to be a.s. Lipschitz continuous in $\theta$, the derivative and expectation cannot be interchanged and the IPA estimator $L^{\prime}(\theta, \omega)$ is biased. In the presence of discontinuities, Smoothed Perturbation Analysis (SPA) can be applied conditioning on the trajectories where jumps occur for small perturbations of size $\Delta \theta$. Two approaches are covered here. First, replacing $L(\theta)$ by another random variable which is a conditional expectation, say $Z(\theta, \omega)=\mathrm{E}[L \mid \mathcal{G}]$ may result in a Lipschitz continuous "conditional performance", for which IPA is applicable. For Example 1, conditional expectation can be used to express $\mathrm{E}(\tau)=\mathrm{E}\left[N\left(T_{\tau}\right)\right]=\mathrm{E}\left[N\left(T_{\tau}\right) \mid T_{\tau}\right]=$ $\theta \mathrm{E}\left(T_{\tau}\right)$, which follows from Wald's identity. Now the pathwise functional $T_{\tau}(\omega)$ is Lipschitz continuous and the Lipschitz constant has bounded expectation. The effect of conditioning is to integrate such discontitnuities, "smoothing" out the sample performance, hence the name of the method.

The second approach conditions on critical events that result in jumps and proceeds to establish a pathwise analysis for Lipschitz continuity of the trajectories on the conditioning sets, as illustrated in Example 2. 
Example 2. Inventory Model.

Consider a standard periodic review inventory model with backlog. Consecutive demands $\left\{D_{n}\right\}$ are assumed continuous, so that the inventory level $X_{t}$ is the right-continous piecewise linear process of Figure 3, and the embedded chain $\left\{X_{n}\right\}$ at the review epochs is Markovian, $X_{0}=0$ and for $n \geq 0$ :

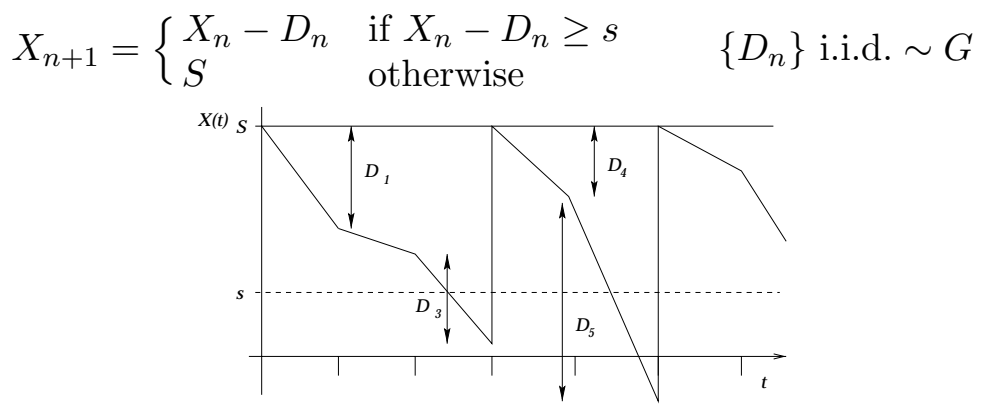

Figure 3 A trajectory of $X_{t}$.

It costs $h$ dollars per unit of inventory at the end of the period, if this quantity is non-negative. Backlog is assumed, with a penalty cost of $p$ dollars per unit of unsatisfied demand. Therefore the cost satisfies:

$$
C_{n}(S) \equiv C\left(X_{n}, D_{n}\right)= \begin{cases}h\left(X_{n}-D_{n}\right) & \text { if } D_{n}<X_{n}-s \\ h\left(X_{n}-D_{n}\right)+K & \text { if } X_{n}-s<D_{n}<X_{n} \\ p\left(D_{n}-X_{n}\right)+K & \text { if } D_{n}>X_{n}\end{cases}
$$

The process is Harris recurrent and bounded in $(s, S)$ so it possesses an ergodic stationary measure. The average long term cost per period is:

$$
J(s, S)=\mathrm{E}\left[L\left(X_{t}, t \geq 0\right)\right]=\lim _{N \rightarrow \infty} \frac{1}{N} \sum_{n=1}^{N} \mathrm{E}\left(C_{n}\right) .
$$

The problem is to find an estimator of the stationary sensitivity:

$$
\frac{\partial}{\partial S} J(s, S)=\lim _{N \rightarrow \infty} \frac{1}{N} \sum_{n=1}^{N} \frac{\partial}{\partial S} \mathrm{E}\left[C\left(X_{n}, D_{n}\right)\right] .
$$

Stochastic Derivative: Define $(\Omega, \mathbb{P})$ as a common probability space where the demands are defined, and let $\mathfrak{f}_{n}=\sigma\left(D_{1}, \ldots, D_{n}\right)$. Then $\left\{X_{n}\right\}$ is a Markov process on $\left(\Omega, \mathbb{F}=\left\{\mathfrak{\mho}_{n}\right\}, \mathbb{P}\right)$, and $X_{n}$ is $\mathfrak{\mho}_{n-1}$-measurable.

For any demand value $d$, the function $C(\cdot, d)$ is continuous, except at $x=d+s$, so that the sample performance $L(\cdot)$ is a Lipschitz continuous function of the process $X_{t}$ in the sup-norm (two proceses $X_{t}$ and $X_{t}^{\prime}$ with $\left\|X-X^{\prime}\right\|_{\infty}<\epsilon$ are shown below). The IPA derivative is: 


$$
\begin{aligned}
& \frac{\partial}{\partial S} C\left(X_{n}, D_{n}\right)=h \mathbf{1}_{\left\{X_{n}>D_{n}\right\}}+p \mathbf{1}_{\left\{X_{n}<D_{n}\right\}} \\
& \frac{1}{N} \sum_{n=1}^{N} \frac{\partial}{\partial S} C\left(X_{n}, D_{n}\right)=\frac{h N^{+}+p N^{-}}{N},
\end{aligned}
$$

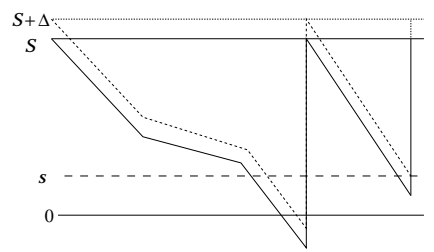

which is not consistent for the derivative, because the process $X_{t}$ is not a.s. Lipschitz continuous in $S$. To see this, let $\tau^{+}(\omega)=\min \{n>1$ : $\left.X_{n}-s<D_{n} \leq X_{n}+\Delta-s\right\}$. Then for all $\omega$ with $\tau^{+}(\omega)<N$, it holds that $\sup _{t \in[0, N)}\left|X_{T}(S+\Delta)(\omega)-X_{t}(S)(\omega)\right| \geq S-s-\Delta \neq \mathcal{O}(\Delta)$.

Identifying the critical events: Define the critical events as $\Omega_{n}=$ $\left\{\omega: \tau^{+}(\omega)=n\right\}$, and express $\Omega$ as the union of disjoint sets $\Omega=$ $\Omega^{*} \cup\left(\cup_{n \leq N} \Omega_{n}\right)$. The set $\Omega^{*}$ is set where the trajectories are Lipschitz

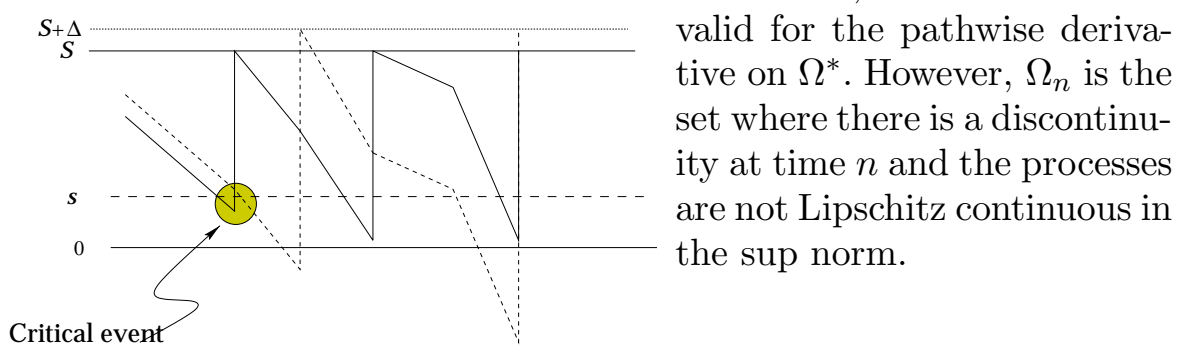

From the assumption that $D_{i}$ has a bounded density, it follows that that critical events are rare in the sense that: $\mathrm{P}\left(\Omega_{n} \mid \mathfrak{\mho}_{n-1}\right)=\mathrm{P}\left(\Omega_{n} \mid X_{n}\right)=$ $\mathcal{O}(\Delta)$. The so-called RPA method ("Rare Perturbation Analysis") was conceived to deal with this type of problem, where the control variable of the DES is of a threshold type (also called structural parameter, in constrast to Example 1, where $\theta$ is a parameter of the continuous lifetime distributions). For state dependent thresholds the "rarity" of the critical events is defined in terms of a conditional expectation, given $\mathfrak{F}_{n}$. This is the typical setting of the SPA method.

The filtered sensitivity: Write the derivative as the limit of a finite difference, $\frac{\partial}{\partial S} J(s, S)=\lim _{N \rightarrow \infty} \lim _{\Delta \rightarrow 0} D(\Delta)$, where:

$$
\frac{D(\Delta)}{\Delta}=\frac{1}{N} \sum_{n=1}^{N} \mathrm{E}\left[\frac{C_{i}(S+\Delta)-C_{i}(S-\Delta)}{\Delta}\right],
$$

and use the law of total probability to express:

$$
\mathrm{E}\left[\frac{D(\Delta)}{\Delta}\right]=\mathrm{E}\left\{\mathrm{E}\left[\frac{D(\Delta)}{\Delta} \mid \Omega^{*}\right] \mathrm{P}\left(\Omega^{*}\right)+\sum_{k=1}^{N} \frac{\mathrm{E}\left[D(\Delta) \mid \Omega_{k}\right]}{\Delta} \mathrm{P}\left(\Omega_{k}\right)\right\} .
$$


The pathwise analysis is now performed on the conditioning sets: for every $\omega \in \Omega^{*}, D(\Delta) / \Delta=\left(h N^{+}+p N^{-}\right) / N+\mathcal{O}(\Delta)$, and $\mathrm{P}\left(\Omega^{*}\right)=$ $1-\mathcal{O}(\Delta)$. On the other hand, for each $k$ and each $\omega \in \Omega_{k}, D(\Delta) \neq$ $\mathcal{O}(\Delta)$, but $\mathrm{P}\left(\Omega \mid \mathfrak{J}_{k}\right)=\mathcal{O}(\Delta)$. Pathwise analysis is used to establish the integrability conditions for a.s. Lipschitz continuity of the product of the conditional expectation and the critical rates:

$$
\lim _{\Delta \rightarrow 0} \sum_{k=1}^{N} \mathrm{E}(\frac{1}{N} \sum_{i=1}^{N} \mathrm{E}[\underbrace{C_{i}(S+\Delta)-C_{i}(S) \mid \Omega_{k}}_{\text {difference process }}] \times \underbrace{\frac{\mathrm{P}\left[\Omega_{k} \mid \mathfrak{\mho}_{k-1}\right]}{\Delta}}_{\text {critical rates }}) .
$$

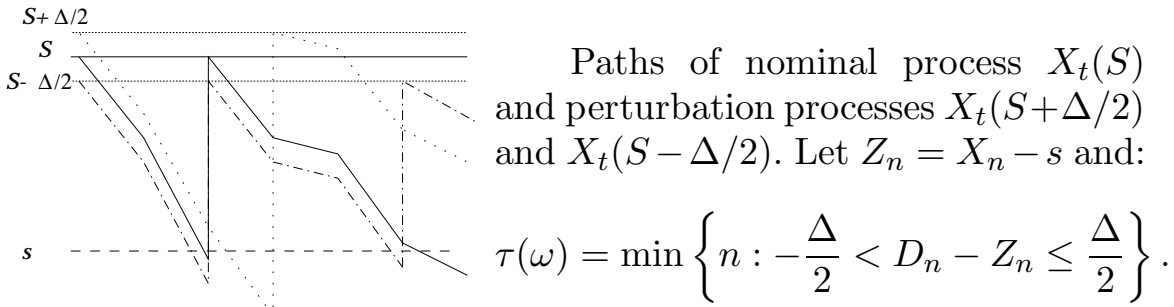

Difference processes: Each trajectory of the perturbed process on $\Omega_{k}=\{\omega: \tau(\omega)=k\}$ coincides with the nominal up to period $k$, where the first discontinuity occurs. At that epoch, reordering happens for one of the processes, which jumps to $X_{k}=S$, while for the other process, the limit state is $X=s$ (no reordering). The number of periods until both process re-order (and thus reach $S$ ) can be bounded by a geometric random variable with finite moments. Therefore, using the Dominated Convergence Theorem, the difference process itself is shown to have a unformly bounded expectation (in $N$ ).

For each $k$, let $X_{n}^{ \pm}(k)$ be the process satisfying $X_{n}^{ \pm}(k)=X_{n}, n<k$, $X_{k}^{-}(k)=S, X_{k}^{+}(k)=s$, and for $n>k$, use the same demands $D_{n}, n>k$ from each trajectory $\omega$ to drive the inventory processes $X_{n}^{ \pm}(k)$. Then $\mathrm{E}\left[D(\Delta) \mid \Omega_{k}\right]=\mathrm{E}\left[L\left(X^{+}(0)\right)-L\left(X^{-}(0)\right)\right]<\infty$ is independent of $\mathfrak{r}_{k-1}$.

Critical rates: As $\Delta \rightarrow 0$, the critical rates satisfy:

$$
\frac{\mathrm{P}\left[\Omega_{n} \mid \mathfrak{\mho}_{n-1}\right]}{\Delta}=\frac{\mathrm{P}\left[X_{n}-\frac{\Delta}{2}-s<D_{n} \leq X_{n}+\frac{\Delta}{2}-s \mid \mathfrak{\mho}_{n-1}\right]}{\Delta} \rightarrow g\left(X_{n}-s\right),
$$

yielding the formula as the sum of the so-called "IPA" and "SPA" terms:

$$
\begin{aligned}
\frac{\partial}{\partial S} J(s, S) & =\lim _{N \rightarrow \infty} \mathrm{E}\left(\frac{h N^{+}+p N^{-}}{N}\right)+ \\
& +\lim _{N \rightarrow \infty} \mathrm{E}\left(\frac{1}{N} \sum_{n=1}^{N} g\left(X_{n}-s\right) \mathrm{E}\left[L\left(X^{+}(0)\right)-L\left(X^{-}(0)\right)\right]\right) \underset{\ldots}{*}
\end{aligned}
$$


The pathwise analysis can be stated in general as follows. A representation $(\Omega, \mathfrak{\jmath}, \mathbb{P})$ is chosen to define an underlying process which is indepedent of $\theta$, and thus

$$
J(\theta)=\int_{\Omega} L(\theta, \omega) \mathbb{P}(d \omega) .
$$

In DES applications, usually the representation is obtained with the Skorohod representation for either the clocks or the state transition functions individually. If there exists $\mathcal{G} \subset \mathfrak{F}$ such that $Z(\theta, \omega)=\mathrm{E}[L(\theta) \mid \mathcal{G}](\omega)$ satisfies the Lipschitz continuity condition, then

$$
J^{\prime}(\theta)=\int_{\Omega} Z^{\prime}(\theta, \omega) \mathbb{P}(d \omega) .
$$

Pathwise analysis entails a deterministic study of the dependency of trajectories in $\theta$, and it is the mechanism by which Lipschitz continuity can be shown. Because the Lipschitz constant must be uniformly integrable in $\theta$, the interchange between derivatives and expectations can only be performed under some integrability assumptions for the DES process itself. Different representations and different conditioning filtrations yield different estimators. Such is the case of the Standard Clock technique that uses a uniformization argument to represent the DES process when the control is a parameter of event lifetimes distributions.

A myriad of methods have been proposed to "extend" the applicability of IPA, such as SPA, SIPA, DPA, RPA and APA. These methods assume particular dependencies on $\theta$ and deal with the resulting discontinuities in an appropriate manner. Ultimately, however, they all make use of the same methodology: showing a.s Lipschitz continuity via a pathwise analaysis and the Dominated Convergence Theorem.

\section{WEAK DIFFERENTIATION}

The dual view to the sample path analysis is called the "weak differentiation" approach, since it uses weak topology concepts. Suppose that the sample performance is a state dependent performance over a finite horizon of a controlled DES $\left\{Z_{n}(\theta), n=1, \ldots, d\right\}$. Let $S=\mathbb{R}^{d}$, then the induced probability measure $\mathrm{P}_{\theta}$ on $(S, \mathcal{B}(S))$ contains all the dependency in $\theta$, so that:

$$
J^{\prime}(\theta)=\frac{d}{d \theta} \int_{S} L\left(z_{1}, \ldots, z_{d}\right) \mathrm{P}_{\theta}(d z)=\int_{S} L\left(z_{1}, \ldots, z_{d}\right) \frac{d}{d \theta} \mathrm{P}_{\theta}(d z) .
$$

It is of course necessary to define what is meant by the derivative of a measure. This will be accomplished using the concept of weak convergence, which gives the name to the method of weak differentiation. Denote by $C_{b}(S)$ be the set of bounded continuous mappings $g: S \rightarrow \mathbb{R}$. 
Definition 15 Let $\mu_{\theta}$ be a family of probability measures defined on $(S, \mathcal{B}(S))$. The probability measure $\mu_{\theta}$ is said to be weakly differentiable if there exists a measure $\mu_{\theta}^{\prime}: \mathcal{B}(S) \rightarrow \mathbb{R}$ such that for every $g \in C_{b}(S)$

$$
\lim _{\Delta \theta \rightarrow 0} \frac{1}{\Delta \theta}\left(\int_{S} g(s) \mu_{\theta+\Delta \theta}(d s)-\int_{S} g(s) \mu_{\theta}(d s)\right)=\int_{S} g(s) \mu_{\theta}^{\prime}(d s) .
$$

It follows that if $\mu_{\theta}^{\prime}$ is the weak derivative of $\mu_{\theta}$ then necessarily $\frac{\partial}{\partial \theta} \mathrm{E}[g]=\int g(s) \mu_{\theta}^{\prime}(d s)$. The difference measure $\mu_{\theta+\Delta \theta}(\cdot)-\mu_{\theta}(\cdot)$ is no longer a probability measure, but rather a signed measure (see Definition 5), satisfying $\mu_{\theta+\Delta \theta}(S)=\mu_{\theta}(S)=1$, and the weak limit (if it exists) is also a finite signed measure with total mass zero.

Example 3. State Dependent Threshold.

Consider a state dependent threshold problem of the general form:

$$
J^{\prime}(\theta)=\frac{d}{d \theta} \int h_{\theta}(x) F(d x), \quad h_{\theta}(x)= \begin{cases}h(x) & \text { if } x<\theta \\ H & \text { otherwise }\end{cases}
$$

where $F$ is the distribution function of $X \in \mathbb{R}^{+}$, assumed to have a bounded density $f$. This representation is in the form suited for pathwise analysis, because the random variable $X$ is independent of $\theta$. Choose for example the representation $X(\omega)=F^{-1}(\omega)$ for $\Omega=[0,1]$.

The dual approach rewrites the integral in terms of:

$$
J^{\prime}(\theta)=\frac{d}{d \theta} \int h(y) F_{\theta}(d y), \quad F_{\theta}(y)=F(y) \mathbf{1}_{\{y<\theta\}}+(1-F(\theta)) \mathbf{1}_{\{y=*\}},
$$

where the support of the distribution is $S=(0, \theta) \cap *$, and one defines $h(*)=H$. This is a mixed distribution, representing a random variable $Y$ as a truncation of the random variable $X$. For any function $g \in C_{b}(S)$,

$$
\begin{aligned}
\frac{d}{d \theta} \int_{S} g(y) F_{\theta}(d y) & =\frac{d}{d \theta} \int_{0}^{\theta} g(x) f(x) d x+g(*)(1-F(\theta)) \\
& =g(\theta) f(\theta)-g(*) f(\theta) .
\end{aligned}
$$

This corresponds to a measure defined for all $B \subset \mathcal{B}(S)$ by the expression $\mu_{\theta}^{\prime}(B)=f(\theta)\left[\mathbf{1}_{\{\theta \in B\}}-\mathbf{1}_{\{* \in B\}}\right]$.

Even if we can identify a signed measure $\mu_{\theta}^{\prime}$ that satisfies the weak differentiation formula, it leaves us with the problem of how to sample from it. In the literature, various approaches have been proposed to transform the above expression into an expression which can be sampled efficiently and we now proceed to cover the basic principles. 
Theorem 4 (Hahn-Jordan Decomposition) For any finite signed measure $\nu$ on $(S, \mathcal{B}(S))$ there exist disjoint sets $S^{+}, S^{-}$such that $S^{+} \cup S^{-}=S$, with the property that for each $A \subset S^{+}, \nu(A)>0$ and for each $A \subset S^{-}, \nu(A)<0$.

Using the Hahn-Jordan decomposition, a weak derivative can be expressed as $\mu_{\theta}^{\prime}(\cdot)=\nu_{\theta}^{+}(\cdot)-\nu_{\theta}^{-}(\cdot)$, where $\nu_{\theta}^{ \pm}(\cdot)$ is the restriction of the measure $\mu_{\theta}^{\prime}$ to the positive and negative sets respectively, defined by $\nu_{\theta}^{ \pm}(B)=\mu_{\theta}^{\prime}\left(B \cap S^{ \pm}\right)$for every $B \subset \mathcal{B}(S)$. Notice now that $\mu_{\theta}^{\prime}(S)=0$ implies that $\mu_{\theta}^{\prime}\left(S^{+}\right)=-\mu_{\theta}^{\prime}\left(S^{-}\right)$, or:

$$
c_{\theta}=\nu_{\theta}^{+}(S)=-\nu_{\theta}^{-}(S),
$$

so that the measures $\mu_{\theta}^{ \pm}(\cdot)$ defined for every $B \subset \mathcal{B}(S)$ by $\mu_{\theta}^{ \pm}(B)=$ $\nu_{\theta}^{ \pm}(B) / c_{\theta}$, are probability measures on $\mathcal{B}(S)$. They are called the (normed) positive and negative parts of $\mu_{\theta}^{\prime}$, respectively. Using these probabilities, the derivative of an expectation can be expressed as the difference of two expectations.

Definition 16 Let $\mu_{\theta}$ be a family of measures on $(S, \mathcal{B}(S))$. A triple $\left(c_{\theta}, \mu_{\theta}^{+}, \mu_{\theta}^{-}\right)$consisting of a positive constant and two probability measures is called a weak derivative of $\mu_{\theta}$ if $\mu_{\theta}$ is differentiable at each $\theta$ and for all continuous bounded functions $g \in C_{b}(\mathbb{R})$ it holds that

$$
\int_{S} g(s) \mu_{\theta}^{\prime}(d s)=c_{\theta}\left(\int_{S} g(s) \mu_{\theta}^{+}(d s)-\int_{S} g(s) \mu_{\theta}^{-}(d s)\right) .
$$

If the left-hand side equals zero for all $g$, we define the weak derivative of $\mu_{\theta}$ as $\left(1, \mu_{\theta}, \mu_{\theta}\right)$.

REMARK: If $\mu_{\theta}$ is weakly differentiable then by definition, $J^{\prime}(\theta)=$ $\int L(s) \mu_{\theta}^{\prime}(d s)$ whenever $L \in C_{b}(S)$. If $L(\cdot)$ is discontinuous but the discontinuity sets $A \subset S$ satisfy $\mu_{\theta}^{ \pm}(A)=0$, then the identity is still true. Unbounded perfomance functions, even if they are integrable, need special care and again the Dominated Convergence Theorem may be used to show case by case what are the appropriate integrability conditions.

In Example 3, $J^{\prime}(\theta)=f(\theta) \mathrm{E}\left[h\left(Y^{+}\right)-h\left(Y^{-}\right)\right]$, with $Y^{+}=\theta, Y^{-}=*$, so that $c_{\theta}=f(\theta)$, and $\mu_{\theta}^{ \pm}$are degenerate measures concentrated at the points $\theta$ and $H$, respectively.

EXAMPLE 2. Inventory Model (revisited.)

A straightforward argument can be used to establish that the SPA derivative w.r.t. $S$ is the negative of the derivative w.r.t $s$ : indeed the process evolution is determined only by $(S-s)$, but the cost depends 
continuously only on $S$ and not on $s$. Writing the expectation in terms of a one step integration:

$$
\mathrm{E}\left(L\left(X_{n+1}, \ldots\right) \mid \mathfrak{\Im}_{n-1}\right)=\int_{0}^{X_{n}-s} \mathrm{E}\left[L\left(X_{n}-y, X_{n+2}, \ldots\right)\right] g(y) d y
$$

where $g$ is the density of the demands and the integral is $\mathfrak{\Im}_{n-1}$-measurable as a function of $X_{n}$. Identify now $G_{s}(y)$ with the truncated distribution:

$$
G_{s}(y)=G(y) \mathbf{1}_{\left\{y<X_{n}-s\right\}}+\left(1-G\left(X_{n}-s\right)\right) \mathbf{1}_{\{y=S-s\}}
$$

and use the chain rule for differentiation on the product of Markov kernels. For the one-step transition expectation and any bounded, continuous, $\mathfrak{r}_{n}$-measurable function $h\left(X_{n+1}\right)$,

$$
\begin{aligned}
\frac{\partial}{\partial s} \mathrm{E}\left[h\left(X_{n+1}\right) \mid \mathfrak{\mho}_{n-1}\right]= & \frac{\partial}{\partial s} \int_{0}^{X_{n}-s} h\left(X_{n}-y\right) g(y) d y+h(S)\left[1-G\left(X_{n}-s\right)\right] \\
& =-g\left(X_{n}-s\right) h(s)+h(S) g\left(X_{n}-s\right) .
\end{aligned}
$$

Choose now $h\left(X_{1}, \ldots, X_{n}, X_{n+1}\right)=\mathrm{E}\left[L(X) \mid \mathfrak{\jmath}_{n}\right]$, then each of the terms in the chain rule differentiation formula now yields the contribution from the one-step transition: $-g\left(X_{n}-s\right) \mathrm{E}\left[L\left(X^{+}(0)\right)-L\left(X^{-}(0)\right)\right]$, recovering the SPA formulation from before.

In many situations it may not be as easy to identify the triple $\left(c_{\theta}, \mu_{\theta}^{+}, \mu_{\theta}^{-}\right)$. In addition, the normed positive and negative parts of $\mu_{\theta}^{\prime}$ are but one of the possible representations for a weak derivative. We now restrict our attention to a specific class of problems for which the family of probability measures $\left\{\mu_{\theta}, \theta \in \Theta\right\}$ possesses a parametrized (differentiable) density and calculation of the derivatives can be done with the standard rules of calculus. This condition is called "absolute continuity". Next, two approaches will be presented that can be helpful in constructing sensitivity estimators, although they both require absolute continuity and are less general than the weak derivative formulation.

Definition 17 A measure $\mu$ on $(S, \mathcal{B}(S))$ is said to be absolutely continuous w.r.t. a measure $\nu$ on $(S, \mathcal{B}(S))$, denoted by $\mu_{\theta}<<\nu$, if for every $B \subset \mathcal{B}(S) \nu(B)=0$ implies $\mu(B)=0$. A family of measures $\mu_{\theta}, \theta \in \Theta$ is said to be dominated by $\nu$ if $\mu_{\theta}<<\nu$, for all $\theta \in \Theta$.

In other words, $\mu<<\nu$ if and only if the support of the distribution induced by $\mu$ (the minimal set $B \subset S$ such that $\mu(B)=1$ ) is contained in the support of the distribution induced by $\nu$. 
Theorem 5 (Radon-Nikodym) Two $\sigma$-finite measures on $\mathcal{B}(S)$ satisfy $\mu<<\nu$ if and only if there exists a nonnegative measurable function on $(S, \mathcal{B}(S))$ denoted by $\left[\frac{d \mu}{d \nu}\right]: S \rightarrow \mathbb{R}$ such that for every $B \in \mathcal{B}(S)$

$$
\mu(B)=\int_{B}\left[\frac{d \mu}{d \nu}\right](s) \nu(d s) .
$$

The function $\left[\frac{d \mu}{d \nu}\right]$ is called the Radon-Nikodym derivative and sometimes it is also called the " $\nu$-density" of $\mu$. For the particular case where $\mu$ is a probability measure on $S \subset \mathbb{R}$ and $\nu$ is the Lebesgue measure, there exists a a measure space $(\Omega, \mathfrak{F})$ where a random variable $X(\omega)$ can be defined such that $X \sim \mu$, and the Radon-Nikodym Theorem states the existence of a Lebesgue density $f_{X}(s): S \rightarrow \mathbb{R}$ such that for any bounded and continuous function $g$,

$$
\mathrm{E}[g(X)]=\int_{S} g(s) f_{X}(s) d s
$$

so that the Radon-Nikodym derivative is the derivative of the distribution.

When both $\mu$ and $\nu$ are probability measures, interpret the RadonNikodym derivative as a random variable on a common probability space $(\Omega, \mathfrak{f})$ where both $X \sim \mu$ and $Y \sim \nu$ are defined. That is, define the nonnegative $\sigma(Y)$-measurable random variable $L(\omega)=\left[\frac{d \mu}{d \nu}\right](Y(\omega))$. This is what is called the Likelihood Ratio.

Definition 18 A $\nu$-dominated family of probability measures $\mu_{\theta}$ is called $L^{1}(\nu)$-differentiable with respect to $\theta$ if the $\nu$-density of $\mu_{\theta}$, denoted by $f_{\theta}$, is $\nu$-almost surely differentiable as function in $\theta$, and both $f_{\theta}$ and $\frac{d}{d \theta} f_{\theta}=f_{\theta}^{\prime}$ are uniformly bounded in $S \times \Theta$.

If $\left\{\mu_{\theta}, \theta \in \Theta\right\}$ is $L^{1}(\nu)$-differentiable family of probability measures on $(S, \mathcal{B}(S))$, then it follows from the Dominated Convergence Theorem that for all $g \in C_{b}(\mathbb{R})$ :

$$
\frac{d}{d \theta} \int g(s) \mu_{\theta}(d s)=\int g(s) \frac{d}{d \theta} f_{\theta}(s) \nu(d s) .
$$

$L^{1}(\nu)$-differentiablility also implies that $\mu_{\theta}^{\prime}$ is absolutely continuous with respect to a measure $\mu_{\theta_{0}}$ for $\theta_{0} \in \Theta$. This allows for the following transformation

$$
\begin{aligned}
\int g(s) \frac{d}{d \theta} f_{\theta}(s) \nu(d s) & =\int g(s)\left(\frac{f_{\theta}^{\prime}(s)}{f_{\theta_{0}}(s)}\right) f_{\theta_{0}}(s) \nu(d s) \\
& =\int g(s)\left(\frac{f_{\theta}^{\prime}(s)}{f_{\theta_{0}}(s)}\right) \mu_{\theta_{0}}(d s)
\end{aligned}
$$


for all $g \in C_{b}(\mathbb{R})$. Evaluating the preceding expression at $\theta_{0}=\theta$,

$$
\frac{d}{d \theta} \int g(s) \mu_{\theta}(d s)=\int g(s) \frac{d}{d \theta} \ln \left(f_{\theta}(s)\right) \mu_{\theta}(d s) .
$$

The mapping $S(\theta, s)=\frac{d}{d \theta} \ln \left(f_{\theta}(s)\right)$ is called the Score Function and the resulting unbiased estimator $g S(\theta)$ is called the Likelihood-Ratio estimator (LR). Notice that the likelihood ratio that apears in the development of the formulas is the ratio $\left(f_{\theta} / f_{\theta_{0}}\right)$, which becomes $\left(f_{\theta}^{\prime} / f_{\theta_{0}}\right)$ upon differentiation w.r.t. $\theta$. However, $\theta_{0}$ is replaced afterwards by $\theta$ itself: any other $\theta_{0}$ can actually serve to define a sensitivity estimator, and in some cases the variance can be reduced choosing $\theta_{0} \neq \theta$.

\section{EXAMPLE 4. Controlled DES.}

It is simpler to see how the LR method works using the general description of $\left\{Z_{n}(\theta)\right\}$ as a homogeneous Markov process in a general state space $S \subset \mathbb{R}^{d}$. Suppose that the transition kernels $P_{\theta}(z, A)=\mathrm{P}\left(Z_{n+1} \in\right.$ $\left.A \mid Z_{n}=z\right)$ are a dominated family with Lebesgue densities $f_{\theta}(z, x)$. Suppose also that the performance function is $L\left(Z_{1}, \ldots, Z_{T}\right) \in C_{b}(S)$. Then a simple application of the chain rule for differentiation yields:

$$
\begin{aligned}
J^{\prime}(\theta)= & \frac{d}{d \theta} \int g\left(s_{1}, \ldots, s_{T}\right) \prod_{i=1}^{T} P_{\theta}\left(d s_{i}, s_{i-1}\right) \\
= & \sum_{j=1}^{T} \int g\left(s_{1}, \ldots, s_{T}\right) \frac{d}{d \theta} \ln \left(f_{\theta}\left(s_{j}, s_{j-1}\right)\right) \times \\
& \prod_{i=j+1}^{T} P_{\theta}\left(d s_{i}, s_{i-1}\right) P_{\theta}\left(d s_{j}, s_{j-1}\right) \prod_{i=1}^{j-1} P_{\theta}\left(d s_{i}, s_{i-1}\right),
\end{aligned}
$$

for any $g \in C_{b}(S)$, which leads to unbiased gradient estimators through the identity:

$$
\frac{d}{d \theta} \mathrm{E}\left[L\left(Z_{1}, \ldots, Z_{T}\right)\right]=\sum_{j=1}^{n} \mathrm{E}\left[L\left(Z_{1}, \ldots, Z_{T}\right) \frac{d}{d \theta} \ln \left(f_{\theta}\left(Z_{j}, Z_{j-1}\right)\right)\right]
$$

The above formulas can again be extended to the case where $T$ is a random stopping time, under appropriate integrability conditions.

REMARK: For the simple model in Example 3, letting $\bar{\theta}=\max (\theta \in$ $\Theta)$ and $\nu$ the corresponding mixed distribution at value $\bar{\theta}$, the RadonNikodym derivatives are: 


$$
\frac{d \mu_{\theta}}{d \nu}(y)= \begin{cases}1 & y \leq \theta \\ 0 & \theta<y \leq \bar{\theta} \\ \frac{1-F(\theta)}{1-F(\theta)} & y=*\end{cases}
$$

which yields an unbounded Score Function.

The weak derivative of a $L^{1}(\nu)$-differentiable measure $\mu_{\theta}$ can be constructed as follows. Let $f_{\theta}$ denote the $\nu$-density of $\mu_{\theta}$, then the positive and negative parts of its derivative $\operatorname{are} f_{\theta}^{ \pm}(s):=\max \left(0, \pm \frac{d}{d \theta} f_{\theta}(s)\right)$, for $s \in S$, and the weak derivative is:

$c_{\theta}:=\frac{1}{2} \int_{S}\left|\frac{d}{d \theta} f_{\theta}(s)\right| \nu(d s)$, and $\mu_{\theta}^{ \pm}(B)=\frac{1}{c_{\theta}} \int_{B} f_{\theta}^{ \pm}(s) \nu(d s), B \in \mathcal{B}(S)$.

If $\mu_{\theta}$ is $L^{1}(\nu)$-differentiable, then $\mu_{\theta}$ is weakly differentiable, but the converse is not true.

Example 5. A Train station.

The quantity of interest is the total waiting time of passengers in transfer at a platform in a subway station,

$$
L(\theta)=\sum_{j=1}^{M} \sum_{k=N\left(V_{j-1}\right)+1}^{N\left(V_{j}\right)} P_{k}\left(V_{j}-S_{k}\right),
$$

where $M$ is the total number of trains per day. The train departure process $M(t)$ is renewal with interarrival times $\left\{Y_{j}\right\} \sim G_{\theta}$ of mean $\theta$ and $V_{j}=V_{j-1}+Y_{j-1}$. The passenger arrival process follows a marked renewal process: at epochs $S_{k}$ the $k$-th group of transfer passengers arrives at the platform, and the size of the group is $P_{k}$, independent of $M(\cdot)$ but assumed to depend on $T_{k}=S_{k}-S_{k-1}$. No synchronization is assumed, so that $\mathrm{P}\left\{S_{k} \in\left(V_{N\left(S_{k}\right)+1}-\epsilon, V_{N\left(S_{k}\right)+1}+\epsilon\right)\right\}=\mathcal{O}(\epsilon)$, which holds if $G_{\theta}$ has a bounded density $g_{\theta}$ and $N(\cdot)$ and $M(\cdot)$ are independent processes.

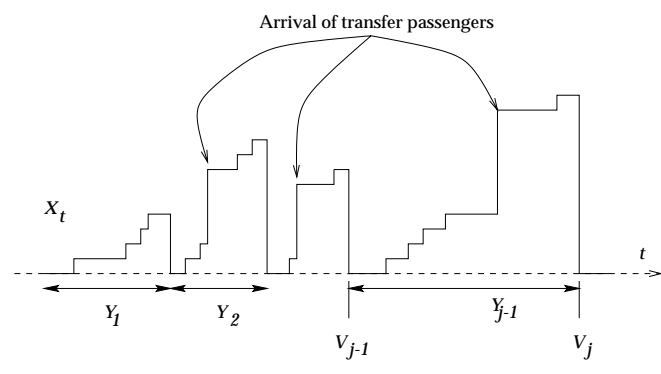

The platform occupancy process $X_{t}$ records the number of passengers waiting at the platform at time $t$, as shown. It is completely described in terms of the model above, and the individual waiting times can be evaluated from the sample paths. 
Under Skorohod representation, let $(\Omega, \mathbb{P})$ be the underlying probability space, and suppose that $Y_{j}=G_{\theta}^{-} 1\left(U_{j}\right)$, for $\left\{U_{i}\right\}$ i.i.d. $\sim U[0,1]$. If $\theta$ is a scale parameter, then $\frac{\partial}{\partial \theta} V_{j}=V_{j} / \theta$. If the function $L(\theta, \omega)$ were Lipschitz continuous in $\theta$ for each trajectory, then the IPA estimator:

$$
L^{\prime}(\theta, \omega)=\frac{1}{\theta} \sum_{j=1}^{M(T)} \sum_{k=N\left(V_{j-1}\right)}^{N\left(V_{j}\right)-1} P_{k} V_{j},
$$

would be unbiased. The discontinuities of this functional are easily described: a negative infinitesimal perturbation of $\theta$ may shift $V_{j}$ so that a particular arrival $k^{*}$ may get into the $j$-th train for the nonperturbed process and wait for train $j+1$ in the perturbed process, thus the difference in contribution to the wait will be of the order $\mathcal{O}\left(V_{j+1}-V_{j}\right) \neq \mathcal{O}(\Delta \theta)$.

From the physical state $X_{t}$, the DES is enlarged to contain information about the two residual clocks: time for the next passenger arrival $R^{1}$ and time remaining for the next train departure, $R^{2}$. Because the model assumes that $P_{k}$ is a function of $T_{k}$ we must enlarge the state with a variable $R^{0}$ to include the appropriate memory. Let the state be $Z_{n}=$ $z=\left(x, r^{1}, r^{2}\right)$, and let $R_{n}^{0}=r^{0}$ be the time since the previous arrival of passengers. If $r^{2}-r^{1}>0$, then the transition probability satisfies:

$$
\mathrm{P}\left[Z_{n+1} \in\left(\{x+m\}, B, r^{2}-r^{1}\right)\right]=\mathrm{P}\left(P_{k}=m \mid T_{k}=r^{0}\right) \int_{B} f(t) d t,
$$

where $f(\cdot)$ is the density of the interarrival times $T_{k}$, and $R_{n+1}^{0}=R_{n+1}^{1}$. All other points have probability zero. On the other hand, if $r^{1}>r^{2}$ then the next event is a train departure, so that $R_{n+1}^{0}=r^{0}$ and:

$$
\mathrm{P}\left[Z_{n+1} \in\left(\{0\}, r^{1}-r^{2}, B\right)\right]=\int_{B} g_{\theta}(t) d t
$$

all other points have probability zero. Let $\mathbb{F}=\left\{\mathfrak{\mho}_{n}, n \in \mathbb{N}\right\}$ be the natural filtration of $\left\{Z_{n}\right\}$. It can be shown that for any $\mathfrak{F}_{n}$-measurable, continuous and bounded function $h\left(Z_{n}\right)$ the transition kernels satisfy:

$$
\begin{aligned}
\frac{\partial}{\partial \theta} \mathrm{E}\left[h\left(Z_{n+1}, \ldots\right) \mid \mathfrak{\mho}_{n}\right]=\mathbf{1}_{\left\{r^{2}>r^{1}\right\}} \times & \\
c_{\theta} & \left(\int \mathrm{E}\left[h\left(\{0\}, R_{n}^{1}-R_{n}^{2}, y\right), Z_{1}, \ldots\right)\right] P_{\theta}^{+}(d y) \\
& \left.\left.-\int \mathrm{E}\left[h\left(\{0\}, R_{n}^{1}-R_{n}^{2}, y\right), Z_{1}, \ldots\right)\right] P_{\theta}^{-}(d y)\right)
\end{aligned}
$$


where $\left(c_{\theta}, P_{\theta}^{+}, P_{\theta}^{-}\right)$is the weak derivative of the distribution of interdeparture times of trains, $G_{\theta}$. The foregoing uses the fact that if the event is a passenger arrival then the derivative of the corresponding transition probability w.r.t. $\theta$ is null. In particular, the performance function is of the form $L\left(Z_{1}, \ldots, Z_{T}\right)=\sum_{n} X_{n}\left|R_{n}^{2}-R_{n}^{1}\right|$, where $T$ is the index corresponding to $M$ train departures.

The estimator is built as follows: every time a train departs, two parallel processes are calculated, say $Z^{ \pm}(k)$ : one for which the next train departure is generated from $g_{\theta}^{+}$and the other where it is generated according to $g_{\theta}^{-}$. If the times between consecutive train departures are exponential with intensity $\theta$, then $g_{\theta}(y)=\theta e^{-\theta x}$ and $g_{\theta}^{\prime}(y)=(1-\theta y) e^{-\theta x}$, with negative and positive densities:

$$
g_{\theta}^{+}(y)=(1-\theta y) e^{-\theta y} 1_{\{0<y \leq 1 / \theta\}}, \quad g_{\theta}^{-}(y)=-(1-\theta y) e^{-\theta y} 1_{\{y>1 / \theta\}},
$$

and $c_{\theta}=e^{-1} /(2 \theta)$. A more realistic model takes account of the fact that trains arriving at the platform come from other platforms in the line and the previous travel times are subject to small random fluctuations. Assuming these fluctuations are i.i.d., at the given platform, trains will be ready for departure every $\theta$ units of time, subject to an approximate normal fluctuation, that is, $Y_{j} \sim \mathcal{N}\left(\theta, \theta^{2} \sigma^{2}\right)$. In this case,

$$
g_{\theta}(y)=\frac{1}{\theta \sigma \sqrt{2 \pi}} \exp \left\{-\frac{1}{2}\left(\frac{y-\theta}{\theta \sigma}\right)^{2}\right\}
$$

and that the weak derivative is calculated with:

$$
\frac{d}{d \theta} g_{\theta}(y)=\frac{1}{\theta}\left(\frac{1}{\theta^{3} \sigma^{3} \sqrt{2 \pi}} y(y-\theta) e^{-\frac{1}{2}\left(\frac{y-\theta}{\theta \sigma}\right)^{2}}-\frac{1}{\theta \sigma \sqrt{2 \pi}} e^{-\frac{1}{2}\left(\frac{y-\theta}{\theta \sigma}\right)^{2}}\right) .
$$

This expression is already the difference between two densities: a doublesided Maxwel density and a normal density (identical to the original one). This weak derivative is not the one corresponding to the normed positive and negative parts of $g_{\theta}^{\prime}$, because their supports are not disjoint. Under this alternative model the processes satisfy $Z^{-}(k) \stackrel{\mathcal{L}}{=} Z$ for all $k$, and only the ds-Maxwell random variable has to be generated to calculate the ensuing performance.

This formulation can be constrasted with the corresponding SPA estimator, which we present without proof. Let $(\Omega, \mathbb{P})$ be an underlying probability space where $Y_{j}=G_{\theta}^{-1}\left(U_{i}\right)$ and $T_{k}$ are defined. Consider the filtration $\mathfrak{\mho}_{k}=\sigma\left(S_{1}, \ldots, S_{k} ; U_{1}, \ldots, U_{M\left(S_{k}\right)-1}\right)$. A critical event $\omega \in \Omega_{k}$ is a trajectory for which the next train departure happens before $S_{k}$ in the path with negative perturbation at $\theta-\Delta \theta$ and after $S_{k}$ in the path calculated at $\theta+\Delta \theta$, as shown below. 
It can be shown that

$$
\frac{\mathrm{P}\left(\Omega_{k} \mid \mathfrak{\Re}_{k}\right)}{\Delta \theta} \rightarrow \frac{S_{k}}{\theta} g_{\theta}\left(S_{k}-V_{M\left(S_{k}\right)}\right)
$$

Also, the difference process satisfies

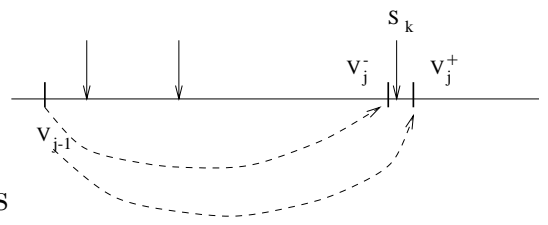
$\mathrm{E}\left[L\left(Z^{+}\right)-L\left(Z^{-}\right) \mid \hat{\mho}_{k}\right]=\theta P_{k}$.

The above yields:

$$
J^{\prime}(\theta)=\mathrm{E}\left[\sum_{j=1}^{M} \sum_{k=N\left(V_{j-1}\right)+1}^{N\left(V_{j}\right)} P_{k}\left(\frac{V_{j}}{\theta}-S_{k} g_{\theta}\left(S_{k}-V_{j-1}\right)\right)\right],
$$

which establishes unbiasedness of the sum of the IPA and the SPA derivative estimators. There is no need for splitting the path when implementing this estimator. Notice, however, that the assumption that $\theta$ is a scale parameter is essential for this estimator to be unbiased, while it is not for the weak derivative estimator, upon the appropriate modifications.

\section{CONCLUDING REMARKS}

Sensitivity estimation for performance measures of DES is a dynamic field of research. Numerous applications have shown that in most interesting cases the sample performances are discontinuous, so that the stochastic derivative, called IPA, is unbiased. Pathwise analysis methods are based on deterministic reasoning to bound the propagation of perturbations. To deal with the different types of discontinuities, a number of extensions of IPA have been proposed. Because it requires explicit construction of an "underlying" probability space, different representations and conditioning can result in very different estimators, and ad hoc arguments and experimentation is done almost case by case. By construction, these derivatives are strongly dependent on the sample performance function itself.

The weak differentiation approach, on the other hand, considers the DES process directly from its formulation in the physical state space. Dealing with space or clock distributions does not change the basic methodology. Because the method is formulated in the weak topology, the estimators are typically valid for all performance measures which are bounded and continuous, or with "rare" discontinuities. In the literature, there are problems where the performance measure is not bounded but satisfies a uniform integrability condition and it has been shown that the weak derivative is unbiased. Establishing general integrability conditions is still an open problem. 
Although we have included explicit examples dealing with finite, infinite and random horizon performances, this course omits the important aspect of the estimation horizon. Finite, infinite and random horizon problems may behave very differently and the integrability conditions may be very difficult to establish for the latter cases. Neither have we included a section on the implementation of the estimators. Clever use of common random variables and stochastic coupling can considerably improve the estimation techniques. For complex and large DES, careful modeling often leads to one preferred formulation, but to date, it seems nearly impossible to identify the models for which one of the available estimation methods performs always better.

\section{Acknowledgements}

This work was partially supported by NSERC grants of the Government of Canada, and partially completed while the author was on leave at the Department of Electrical and Electronic Engineering of the University of Melbourne, Australia. The author wishes to thank Bernd Heidergott, whose insight into weak differentiablility and participation greatly helped in the preparation of this manuscript.

The following list of references is minimal. It is intended to cover the main texts available in the subject, rather than the extended list of references that have necessarily been consulted for the preparation of this course.

\section{References}

[1] P. Billingsley, Convergence of Probability Measures, John Wiley and Sons, New York, 1968.

[2] P. Billingsley, Probability and Measure, John Wiley and Sons, New York, 1979.

[3] C.G. Cassandras and S. Lafortune Introduction to Discrete Event Systems, Kluwer Academic, Boston, 1999.

[4] M. Fu and J.-Q. Hu. Conditional Monte Carlo, Kluwer Academic, Boston, 1997.

[5] P. Glasserman, Gradient Estimation via Perturbation Analysis, Kluwer Academic Publishers, Boston, 1991.

[6] G. Pflug. Optimisation of Stochastic Models, Kluwer Academic, Boston, 1996.

[7] R. Rubinstein and A. Shapiro, Discrete Event Systems: Sensitivity Analysis and Optimization by the Score Function Method. Wiley, 1993. 